Beispiel die Satzung der FU dreimal aufgeführt wird: Einmal unter Satzung (S. 350), einmal unter Bundesgeschäftsstelle (S. 347) und im Text als „Zitiert nach“ (Fußnote 410 auf S. 129). Aussagen von Politikerinnen werden unter „Reden“ ablegt. Daraus folgt im Text ein buntes Nebeneinander von Organisationsmerkmalen, Eigen- und Fremdbeurteilungen ohne Rücksicht auf Entstehungszeit und Kontext. Ähnlich unerfreulich ist die Zitierweise: uneinheitlich, eingerückt, nicht eingerückt, mit und ohne Anführungszeichen, was den teilweisen hohen Anteil von aneinandergereihten Zitaten verdeckt (S. 202).

Es bleibt zu hoffen, dass dieses in fünf Jahren Arbeit nach den im Studium vermittelten Regeln entstandene - und als Dissertation angenommene - Buch nicht den derzeitigen wissenschaftlichen Standard spiegelt.

Ingrid Reichart-Dreyer

\title{
Parteienrecht: Standardwerk mit Schweizer Besonderheiten
}

Schiess Rütimann, Patricia M.: Politische Parteien. Privatrechtliche Vereinigungen zwischen öffentlichem Recht und Privatrecht (Schriften zum Parteienrecht und zur Parteienforschung, Band 41), Nomos Verlagsgesellschaft, Baden-Baden 2011, 808 Seiten, € 134,-(zugleich erschienen im Stämpfli Verlag, Bern 2011, 726 Seiten, CHF 154,-).

Die schwergewichtige Habilitationsschrift von Patricia M. Schiess Rütimann widmet sich dem Thema der Organisation, der Mitgliedschaft und der inneren Willensbildung der politischen Parteien in Belgien und der Schweiz, und zwar mit einem deutlichen Schwerpunkt auf der Schweiz. Das schweizerische Arrangement wird dabei verglichen mit dem Befund in Belgien und das so gewonnene Bild dann in Kontrast gesetzt zu einigen grundlegenden Merkmalen des Parteienrechts in Deutschland. Schiess Rütimann untersucht, inwieweit die in Belgien und vor allem in der Schweiz vorhandenen privatrechtlichen Normierungen sich in den öffentlich-rechtlichen Rahmen einfügen und geeignet sind, den Parteien ein hinreichendes rechtliches Gerüst zu geben oder ob zusätzliche Regelungen notwendig sind. Diese Frage kann aber, wie auch ausdrücklich festgestellt wird (Rn. 27 f.), nicht ohne Einbeziehung der empirischen Ebene beantwortet werden. Das bereits aufwendige rechtsvergleichende Programm reichert die Autorin daher noch an, indem sie politikwissenschaftliche Erkenntnisse zur Analyse heranzieht und daneben selber Tatsachenerhebung betreibt, vor allem durch eine umfangreiche Auswertung der Presse, daneben aber auch durch Interviews mit Parteifunktionären, um die praktische Adäquanz der untersuchten Regulierungen zu überprüfen.

Die Schrift geht in fünf Schritten vor: Im ersten Teil werden Untersuchungsgegenstand, Ziele und Methoden, darüber hinaus auch das verfassungsrechtliche und politische Umfeld der schweizerischen und belgischen Parteien vorgestellt. Die verfassungsrechtliche Lage der Parteien in Deutschland wird kurz gestreift. Der zweite Teil geht ausführlich auf die Organisation und Rechtsform der schweizerischen Parteien ein und behandelt die parteiinterne Gerichtsbarkeit, die Finanzierung und die Kandidatenaufstellung. Der Rolle der Parlamentsfraktionen für die Parteien in Belgien und der Schweiz und der Stellung von Inhabern staatlicher Ämter innerhalb der Parteien wendet sich der dritte Teil zunächst zu, um sich 
dann mit den Mitgliedern und den Aufnahmevoraussetzungen der Parteien beider Länder zu befassen. Im vierten Teil werden wiederum mit großer Akribie zunächst die Entscheidungsorgane und -verfahren der Schweizer Parteien untersucht; den belgischen Parteien wird etwas weniger Aufmerksamkeit gewidmet. Anschließend werden die rechtlichen Sicherungen der innerparteilichen Demokratie untersucht. Bevor die Lage in der Schweiz und in Belgien ins Blickfeld rückt, wird die innerparteiliche Demokratie in Deutschland knapp skizziert, bevor sich die Autorin den sozialwissenschaftlichen Untersuchungen über die tatsächlich gelebte Demokratie in den Schweizer und belgischen Parteien widmet. Im fünften und letzten Teil schließlich fragt sie, ob die Regulierungen in der Schweiz hinreichend seien. Von Änderungsbedarf in Details abgesehen wird diese Frage positiv beantwortet, vor allem benötige die Schweiz kein eigenes Parteiengesetz. Eine Zusammenfassung schließt die eigentliche Untersuchung ab, es folgt mit Blick auf den Schweizer Leser ein Anhang zu belgischen Parteien und den belgischen, teilweise auch deutschen Rechtsgrundlagen.

Bei aller beeindruckenden Detailfülle und Genauigkeit der Untersuchung bleiben doch aus deutscher Sicht gewisse Zweifel bezüglich einzelner Ergebnisse: Ob und inwiefern etwa eine staatliche Regulierung der inneren Ordnung der Parteien für die Schweiz verzichtbar ist (Rn. 1268), bleibt immerhin fraglich. Der Hinweis, die Neugründung einer eigenen Partei durch Mitglieder, die mit undemokratischen Verhältnissen unzufrieden sind, sei rechtlich ohne Schwierigkeiten möglich (Rn. 1217), trifft sicher zu. Politisch ist der Austritt aus einer etablierten und die Gründung einer neuen Partei aber mit erheblichen Kosten verbunden. Ebenso garantiert eine Regelung der Kandidatenaufstellung, welche die Basis gegenüber den Parteivorständen zur Letztentscheidung ermächtigt, zwar per se keinen willkürfreien Wahlvorgang (Rn. 1219), trüge aber doch zu ihm bei. Zudem kommt dem Verfahren der Bestimmung von Kandidaten für staatliche Ämter ein legitimatorischer Eigenwert zu. Ebenso verwundert das Argument, die gesetzliche Garantie einer demokratischen Willensbildung innerhalb der Parteien bedeute deren „Gleichschaltung“ (Rn. 1221 f.). Darum geht es nicht, sondern um eine gegenüber Sach- und Personalfragen neutrale, rechtliche Absicherung des demokratischen Charakters innerparteilicher Entscheidungsvorgänge. Dass der empirische Befund bezüglich innerparteilicher Demokratie in der Schweiz dennoch positiv ausfällt (Rn. 1274 ff.), liegt möglicherweise an Faktoren außerhalb des Parteienrechts (Rn. 1216 ff.; Rn. 1384 ff.).

So ist wohl auch die faktische Verzichtbarkeit eines Parteiengesetzes für die Schweiz zu erklären, die ein Ergebnis der Untersuchung darstellt (Rn. 1308 ff.): Letztlich stand das schweizerische ZGB vor demselben Problem wie das deutsche BGB, nämlich einen rechtlichen Rahmen für eine unübersehbare Vielzahl nach Umfang, Zwecken und Organisationsstruktur verschiedener Vereine zu schaffen. Die Frage ist daher zunächst, ob Vereine mit einer so speziellen Zielsetzung und Bedeutung wie es politische Parteien sind, nicht einer besonderen rechtlichen Regulierung bedürfen oder ob eine solche angepasste Regulierung nicht zumindest eine Reihe von Vorteilen bietet. Auch wenn eine Spezialregelung Vorteile bietet, kann man allerdings je nach politischem Bezugsrahmen ohne sie auskommen. Hier ist es plausibel, dass die Schweiz über eine derartig gefestigte demokratische Politische Kultur verfügt und über so wirksame und etablierte Wege demokratischer Herrschaft, die nicht über die Parteien führen, dass in der Tat besondere rechtliche Sicherungen der innerparteilichen Demokratie oder der finanziellen Transparenz, wie sie etwa das deutsche Parteiengesetz kennt, nicht notwendig sind. Dass allerdings die Gefahr bestünde, solche Garantien schwächten sogar potentiell die innere Demokratie der Parteien (Rn. 1229), erscheint als zu 
weitgehend. Dennoch, der Leser nimmt als wertvolle Anregung mit, sich bei der Beurteilung von Rechtsregimen nicht nur mit den Vorzügen oder Nachteilen der einzelnen Regelungen zu befassen, sondern auch ihre gesellschaftlichen Rahmenbedingungen einzubeziehen.

Trotz der dargelegten Zweifel an einigen Wertungen wird das von Schiess Rütimann vorgelegte Werk zur Information über das belgische und besonders das Schweizer Parteienrecht mit seiner stupenden Informationsfülle wohl auf Jahre hin unverzichtbar sein.

Sebastian Roßner

\section{Das Ende von New Labour: drei Ereignisgeschichten und ein Schuldiger}

Mandelson, Peter: The Third Man, Harper Press, London 2011, 577 Seiten, £ 8,99.

Rawnsley, Andrew: The End of the Party. The Rise and Fall of New Labour, Penguin Books, London 2011, 895 Seiten, £ 12,99.

Seldon, Anthony und Guy Lodge: Brown at 10, Biteback Publishing, London 2011, 520 Seiten, $£ 14,99$.

New Labour - das war zunächst eine Erfolgsstory. Tony Blairs Wahlerfolg von 1997 wurde als Versprechen eines Neuanfangs, als der Beginn von Cool Britannia jenseits traditioneller Politik interpretiert. Was aus diesem Aufbruch wurde, ist heute noch umstritten. Patrick Diamond hat den widersprüchlichen Charakter von New Labour treffend zusammengefasst: „It was influenced by Thatcherism, explicitely rejected the traditional ethos of British social democracy, yet governed as previous Labour administrations had done, resdistributing the fruits of economic growth into public services and the welfare state. "1 Nicht vergessen werden darf, dass diese Politik, die sich selbst jenseits von Rechts und Links verortete, von Anfang an drei Konstruktionsfehler hatte: Sie machte die Labour Party zu einem Verbündeten der Globalisierung zugunsten der Finanzwelt, aber auf Kosten der Wählerschaft der Partei. Sie war unfähig mit den Herausforderungen durch langjährige Kriege (Irak, Afghanistan) umzugehen, und sie litt unter der Doppelregentschaft von Tony Blair und Gordon Brown. Anfangs wurde letztere durch eine Arbeitsteilung der beiden politischen Schwergewichte der Labour Party befriedet, und Gordon Brown konnte davon ausgehen, in absehbarer Zeit Nachfolger Tony Blairs zu werden. Seit 2003 wurde daraus eine offene Schlacht zweier Regierungslager um das Amt des Premierministers.

Was im Einzelnen geschah zeichnen zwei der hier zu betrachtenden Bände in höchster Detailgenauigkeit und auf der Basis von hunderten von Interviews nach. Anthony Seldon hat mit wechselnden Partnern zwischen 1990 und 2010 vier Bücher über die Amtsführung britischer Premierminister veröffentlicht. Er sieht sich der Zeitgeschichte verpflichtet und

1 Patrick Diamond, Governing as New Labour: An Inside Account of the Blair and Brown Years, in: Political Studies Review, 9. Jg. (2011), S. 145 - 162, S. 146. Vgl. auch Roland Sturm, Der „dritte Weg“" Demokratie im Zeitalter der Globalisierung, in: Uwe Backes / Eckhard Jesse (Hrsg.), Jahrbuch Extremismus \& Demokratie, 12. Jg., Baden-Baden 2000, S. 53 - 72. 